\title{
Erste Hilfe im Gehirn: Wenn die Sprache plötzlich ausfällt
}

Hartwigsen G et al. Rapid short-term reorganization in the language network. elife 2017.

doi:10.7554/eLife. 25964

Nach einem Schlaganfall treten bei den Betroffenen häufig Sprachprobleme auf. Manche können bestimmte sprachliche Fähigkeiten wiedererlangen, anderen bleiben sie jedoch für immer verloren. Wissenschaftler des Max-Planck-Instituts für Kognitionsund Neurowissenschaften in Leipzig haben 
nun eine mögliche Erklärung gefunden: Die Verletzung einiger Hirnbereiche kann gut kompensiert werden, die anderer Regionen hingegen nicht. Diese Erkenntnisse könnten nicht nur für die Therapie eines Schlaganfalls relevant sein, sondern bestätigen auch den hierarchischen Aufbau der Sprache.

Die Forscher haben herausgefunden, was passiert, wenn zwei entscheidende Hirnareale für unser Sprachverständnis plötzlich inaktiv sind: Ist der Gyrus angularis beeinträchtigt, in dem die Bedeutung der Sprache verarbeitet wird, kann das Gehirn das gut kompensieren: Der benachbarte Gyrus supramarginalis springt ein und verstärkt seine Aktivität - obwohl dieser eigentlich dafür zuständig ist, die rhythmische Struktur der Wörter zu verarbeiten. Dadurch könne die Bedeutung von Wörtern beinahe genauso schnell erkannt werden, als wenn das eigentlich zuständige Areal diese Aufgabe erfülle. Ist dagegen der Gyrus supramarginalis selbst gestört, kann sein Ausfall kaum kompensiert werden, und seine Aufgaben werden von keinem anderen Teil des Sprachnetzwerkes übernommen. Für Betroffene wird es dann deutlich schwerer, die rhythmische Struktur eines Wortes zu verarbeiten, also seine Silben zu analysieren.

Untersucht haben die Neurowissenschaftler diese Zusammenhänge mithilfe der transkraniellen Magnetstimulation (TMS). In diesem Falle hemmte das Team bei 17 gesunden Studienteilnehmern für kurze Zeit jeweils das Sprachareal für die Analyse der Wortbedeutung oder der rhythmischen Struktur. Daraufhin verglichen sie die Leistungen der Teilnehmer in sprachlichen Aufgaben.

Die Wissenschaftler vermuten, dass die Fähigkeit, einen gestörten Prozess durch einen anderen Hirnbereich zu kompensieren, davon abhängt, auf welcher Hierarchieebene die Sprache gestört wird: Handelt es sich um einen derart grundlegenden Prozess wie die Verarbeitung der rhythmischen Struktur eines Wortes, kann er nicht einfach von anderen Bereichen übernommen werden. Komplexere Verarbeitungsschritte wie die Bedeutungsanalyse können jedoch von einfacheren Prozessen unterstützt werden, da sie auf diesen aufbauen. Allgemeinere Prozesse können dann unterstützend herangezogen werden um so die Verarbeitung aufrechtzuerhalten.

Aus diesen Erkenntnissen schließen die Autoren zweierlei: Zum einen könne man abschätzen, welche Schädigungen sich - etwa nach einem Schlaganfall - am ehesten kompensieren lassen, und man in Zukunft verstärkt die Therapie ausrichten könnte. Zum anderen konnten sie die Hypothese vom hierarchischen Aufbau der Sprache bestätigen: Demnach bauen während der Verarbeitung von Sprache komplexe Schritte auf einfacheren auf. Bevor man also die Bedeutung eines Wortes analysiert, verarbeitet man also zunächst dessen Laute.

Nach einer Mitteilung des Max-Planck-Instituts für Kognitions- und Neurowissenschaften 